\title{
Periódico El Coquillo como estrategia pedagógica para fortalecer el pensamiento crítico de los estudiantes1
}

\section{The newspaper "El coquillo" as a pedagogical strategy to strengthen the critical thinking of students}

DOI: http://dx.doi.org/10.17981/cultedusoc.9.3.2018.70

Artículo de investigación. Fecha de recepción: 15/06/2018. Fecha de aceptación: 27/11/2018

\author{
Arnobis Caro Bazza'; \\ Pedro Ruiz; Nelson Martínez Orozco; \\ Eduardo Padilla Ruiz y Luis Aguilera Rodero ${ }^{3}$ \\ Institución educativa Celinda Mejía López (Colombia) \\ madelinsanchezotero69@gmail.com
}

Para citar este artículo:

Caro, A., Ruiz, P., Martínez, N., Padilla, E. y Aguilera, L. (2018). Periódico El Coquillo como estrategia pedagógica para fortalecer el pensamiento crítico de los estudiantes. Cultura. Educación y Sociedad 9(3), 591-602. DOI: http://dx.doi.org/10.17981/cultedusoc.9.3.2018.70

\section{Resumen}

La lectura es una fuente importante de conocimiento, por lo que es ampliamente impulsada en las escuelas. El propósito de la presente investigación es fortalecer el pensamiento crítico a través del periódico El Coquillo en los estudiantes del grado sexto de la Institución Educativa Celinda Mejía López. Como instrumentos de recolección de la información se emplearon la observación, la encuesta y la entrevista. La mayoría de estudiantes afirman que la variedad en los textos asignados para la lectura incide altamente en su deseo de leer, en cuanto a los tipos de lectura, la narrativa sobresale entre las demás opciones. La variedad influye ampliamente en los procesos de una enseñanza significativa porque a través de esta se despierta el interés de los estudiantes por la lectura y se logra incidir en que adquieran un proceso lector crítico, que es una de las competencias que se evalúan en gran cantidad de pruebas estatales.

Palabras clave: Proceso lector, lectura crítica, conocimiento, enseñanza.

\section{Abstract}

Reading is an important source of knowledge, which is why it is widely promoted in schools. The purpose of this research is to strengthen critical thinking through the newspaper El Coquillo in the sixth grade students of the Educational Institution Celinda Mejía López. As instruments of information collection, observation, survey and interview were used. The majority of students affirm that the variety in the texts assigned for the reading affects highly in their desire to read, as for the types of reading the narrative stands out among the other options. Variety greatly influences the processes of meaningful teaching because through this the student's interest in reading is aroused and it is achieved that they acquire a critical reading process, which is one of the competencies that are evaluated in great quantity. of state tests.

Keywords: Reading process, critical reading, knowledge, teaching.

1 Este artículo ha sido derivado del Programa de Fortalecimiento de la Cultura Ciudadana y Democrática CT+I a través de la IEP apoyada en TIC en el Departamento de Magdalena: CICLON

2 Líder del grupo de investigación "Panel".

3 Docentes de la institución educativa Celinda Mejía López.

- The author; licensee Universidad de la Costa - CUC.

Cultura, Educación y Sociedad vol. 9 no. 3, pp. 591-602. Diciembre, 2018

Barranquilla. ISSN 2389-7724 Online 


\section{Introducción}

Muchos de los estudiantes de la Institución Educativa Celinda Mejía López de Santa Ana Corregimiento de Barroblanco y especialmente los que cursan sexto grado presentan serias dificultades al momento de leer un texto. Este hecho no les permite ejercer la comprensión lectora apropiadamente, avanzar a niveles más altos de interpretación y que sean capaces de desarrollar pensamiento crítico a través de lo que se lee.

Esta problemática se ve reflejada en los resultados en las distintas pruebas tanto internas como externas a la que se someten los educandos periódicamente, obteniendo desempeños bajos en los últimos tres años; de tal forma que afecta el desarrollo cognitivo en las demás áreas del conocimiento, no alcanzando los niveles deseados de aprendizaje propuestos en los programas académicos de la institución, direccionados por el (MEN) Ministerio de Educación Nacional.

Con frecuencia se evidencia que los estudiantes se les dificultan responder las ideas generales de un texto. A duras penas, se dan cuenta, de aspectos locales o de situaciones aisladas que no recoge la universalidad de la historia o el tema planteado por el autor. Se limitan a identificar palabras prescindiendo del significado de estas y no son capaces de asociar ideas o conceptos, y convirtiendo el proceso lector en un acto mecánico en el cual se acude más a la decodificación superficial que a la profundización de contenidos (Morán y Uzcátegui ,2006). Además, en el desarrollo de las distintas actividades, que implica lectura, los estudiantes muestran lentitud para apropiarse y concluir objetivamente las acciones que permiten el progreso de la lectura y el pensamiento crítico (Serrano, 2008).
Las prácticas educativas actuales, difícilmente permiten el desarrollo de competencias que exige la realidad actual, se dificulta promover pensamiento crítico, expresar sus puntos de vista, reflexionar frente a la resolución de problemas, ser autónomos en el aprendizaje y tomar decisiones; idea que corrobora De Zubiría (2014), cuando comenta que la educación básica no se dedica a desarrollar competencias transversales para pensar, convivir, interpretar.

Por otra parte, las instituciones educativas se preocupan por alcanzar un buen nivel académico y se descuida en cierta medida el desarrollo humano, el fortalecimiento de competencias actitudinales que generen autonomía y seguridad, como el saber ser, saber convivir, saber sentir y saber actuar e incluso la posibilidad que tienen de manifestar públicamente (de buenas maneras) sus preferencias individuales y colectivas (por ejemplo) (Valenzuela y Rochin, 2013). Los docentes se centran en el cumplimiento de sus planes de clase, se guían por los contenidos establecidos y generalmente con clases magistrales (Almeida, Coral, Ruiz, y Grisales 2014).

Hoy por hoy, el estudiante normalmente no se encarrila en el conocimiento aprender, tampoco el docente lo hace en su práctica pedagógica. En tal sentido, nuestros niños y jóvenes no abordan la metacognición como una estrategia válida y efectiva para desarrollar su propio aprendizaje y pensamiento, promoviendo de manera autónoma la construcción del conocimiento.

López (2011) conceptúa que los estándares de aprendizaje contemporáneos requieren de una educación que se enfoque en el desarrollo conceptual del pensamiento creativo, de la resolución de problemas y de la formulación y comunicación de argumentos. Que los estándares de evaluación, exigen un cambio en las pruebas 
de las que evalúan la memorización de datos, a las evaluaciones auténticas de desempeño articuladas a las actividades de aprendizaje.

La lectura crítica tiene una gran importancia porque afecta a la población estudiantil, ya que, constituye un gran desarrollo en la forma de comprender, interpretar, criticar y obtener un pensamiento crítico, es decir, aceptar o no las ideas de un autor plasmadas en un texto o un problema que se presente en la cotidianidad (Páez y Rondón, 2014).

Baqueriza y Anchundia (2013) afirman que la lectura crítica es concebida como un proceso activo desencadenado por la acción y estrategia del pensamiento, mediante esto el estudiante realiza una crítica, la forma de realizarlo es modificada y se aproxima a la interacción entre el sujeto y la información.

Las investigaciones sobre el pensamiento y la lectura crítica tienen una gran importancia, ya que la lectura constituye sin duda, la más importante adquisición de saberes para desarrollar la imaginación y la creatividad, además, es fuente de cultura, mejora el manejo de la escritura, entre otras. Por lo tanto, se hace necesario implementar estrategias, que estimulen la lectura en los estudiantes, fomentando un pensamiento crítico y un razonamiento lógico que facilitará el proceso en el aprendizaje con las diferentes áreas y/o asignaturas (López, 2012).

En este sentido, es muy importante asumir un compromiso de desarrollar significativamente la lectura crítica en los estudiantes, puesto que los ayuda a ser más sociales, comprender cualquier tipo de textos, ser críticos entre otras habilidades. Todas estas características se emplearán en la población más necesitada, por lo menos en nuestra área de influencia que en este caso se ubica en la Escuela Normal Superior "La Hacienda", buscando la transformación en los procesos de enseñanza y en la forma de pensar de las nuevas generaciones (Montoya y Monsalve, 2008).

Las investigaciones sobre lectura crítica tienen una gran importancia ya que constituye la más importante adquisición de saberes para desarrollar la imaginación y la creatividad, además, es fuente de cultura, mejora el manejo de la escritura, entre otras.

La lectura crítica depende de la comprensión, del pensamiento, de la imaginación, la memoria, la práctica intima, la estética, la producción de conocimiento al igual que las prácticas sociales y culturales que tenga el individuo en este caso el estudiante (Serrano, 2014). Se debe tener en cuenta el contexto en el que se desenvuelven ellos para mejorar sus competencias y habilidades comunicativas, resaltando sus talentos y evoluciones dentro de esta disciplina (Serrano, 2014).

Por lo tanto, se hace necesario implementar estrategias, que estimulen la lectura en los estudiantes, fomentando un pensamiento crítico y un razonamiento lógico que facilitará el proceso en el aprendizaje con las diferentes áreas y/o asignaturas. Este trabajo está centrado en el análisis y búsqueda del desarrollo del pensamiento crítico para alcanzar mejores niveles de aprendizaje y configurar el conocimiento como un instrumento eficaz para construir una postura reflexiva en la vida cotidiana $\mathrm{y}$ académica.

\section{Pensamiento crítico}

No resulta fácil definir el pensamiento crítico, especialmente cuando existe una diversidad de conceptos, en un gran número de pensadores e investigadores acerca de este tema. Según Tamayo y Tamayo (2003), se define como un pensamiento acertado y reflexivo, orientado en qué pensar y en qué hacer. Requiere llevar a cabo acciones como las siguientes: juzgar la 
credibilidad de las fuentes, identificar las conclusiones, razones y supuestos, juzgar la calidad de un argumento, sus razones, supuestos y evidencias. Refleja una posición independiente a cerca de un asunto, permite hacer preguntas clarificadoras, planificar y diseñar experimentos, definir términos de manera apropiada, tener apertura mental, estar bien informado y sacar conclusiones.

Mendoza (2015), en su tesis La Investigación y el Desarrollo del Pensamiento Crítico en Estudiantes Universitarios, establece la importancia para el desarrollo del pensamiento crítico, en el cual resulta imprescindible en la formación del estudiante universitario, tanto desde el punto de vista profesional como personal; hace una reflexión sobre el papel de la metodología de formación universitaria, basadas en el desarrollo de competencias investigativas y su relación con el desarrollo del pensamiento crítico. Además, este trabajo estudia y analiza la formación del pensamiento crítico en los estudiantes, preparándolos para la vida profesional, y la eficacia de la metodología tradicional frente a las necesidades reales de formación.

Paul y Elder (2003), dicen:

"El pensamiento crítico es ese modo de pensar sobre cualquier tema, contenido o problema en el cual el pensante mejora la calidad de su pensamiento al apoderarse de las estructuras inherentes del acto de pensar y al someterlas a estándares intelectuales" (2003).

\section{Pensar críticamente}

El concepto de Pensamiento Crítico no ha sido definido de una manera precisa, ya que no existe un acuerdo común entre los distintos investigadores sobre el tema (Bailin, Case, Coombs y Daniels, 1999), presentan un resumen de las distintas investigaciones indicando que falta claridad y profundidad didáctica sobre el tema.
Se ha conceptualizado el Pensamiento Crítico como juicio, análisis, opiniones, pensamiento, metacognición, razonamiento y solución de problemas (Arrieta, 2016). Se le ubica como conocimiento, autorregulación mental, habilidad de pensamiento complejo, sin precisar un contexto y contenido.

La literatura compara al Pensamiento Crítico con procesos mentales, pero sin existir unanimidad en una definición de lo que sería pensar críticamente (González, 2008). Citando a Fowler (1997), expone una serie de proposiciones de diversos autores sobre lo que sería "pensar críticamente", observando la cercanía, relación y complementariedad que existe entre ellas.

González (2008), concluye que el Pensamiento Crítico está relacionado con la emisión de buenos juicios y con la forma de pensar de quien desea la verdad. Así mismo, encuentra las características o requerimientos con los que debería contar una persona para pensar críticamente.

Entre ellos tenemos:

a. Comprender y evaluar diferentes puntos de vista.

b. Cuestionar el propio pensamiento.

c. Tener mente abierta y honestidad intelectual.

d. Evaluar y decidir razonada y reflexivamente.

e. Razonar lógica y coherentemente.

\section{Estructura del pensamiento crítico}

Bierman y Assali (1996) han desarrollado un Manual del Pensamiento Crítico que propone una estructuración de este. Según los autores, los argumentos tienen una naturaleza que no depende del contenido sino de las intenciones. Es así, como si las premisas buscan garantizar la conclusión, el argumento se considera deductivo, mientras que, si las premisas sólo buscan dar apoyo el argumento se considera inductivo. 
Para Bierman y Assali (1996) se cometen frecuentemente errores por parte de los pensadores críticos en cuanto a la evaluación de los argumentos deductivos o inductivos. En cuanto a la evaluación de los argumentos deductivos, no se suele evaluar su sensatez y veracidad. Así mismo, al evaluar los argumentos no se consideran criterios como el respeto, el buen uso del lenguaje y la simplicidad.

Respecto a la evaluación de argumentos inductivos Bierman y Assali (1996) sugieren ahondar en la inducción enumerativa, el silogismo estadístico, la inducción diagnóstica y los argumentos estadísticos, lo que conlleva a identificar las conclusiones, a clarificar los términos clave, a simplificar y parafrasear cuando sea necesario, evitando confundir razonamientos inductivos con deductivos, caer en falacias de generalización, en el uso inadecuado de la evidencia, así como en falacias estadísticas, jugadoras y causales.

Santiuste et al., (1996) complementan esta perspectiva planteando que además de la lógica formal, el Pensamiento Crítico procede implícitamente mediante otros aspectos como: escuchar a otros, actuar de distinta manera, pensar autónomamente y anticipar procesos de búsqueda, planteando la existencia de formas de pensamiento no necesariamente lógicas (Lipman et al., 1992), presentan una estructura de tres elementos concurrentes al "Pensar Lógicamente": respetar la lógica formal, dar buenas razones, y actuar racionalmente.

\section{Componentes del Pensamiento Crítico}

Como todo proceso mental, el "pensamiento crítico" para accionar requiere de tres componentes: conocimiento, habilidades y actitudes. a. Conocimientos: El acto de pensamiento nace del conocimiento de aquello en lo que hay que pensar. Aunque la literatura se ha concentrado en las habilidades y disposiciones (McPeck, 1981; Ennis, 1987; Halpern, 1998), no debe dejarse de lado el propio acto cognoscitivo como punto inicial para la ejecución del Pensamiento Crítico, con todas las implicaciones que conlleva el acto cognoscitivo: la percepción, la imaginación, el sentido común, la memoria, intervienen cada vez que se formula y establece un pensamiento.

b. Habilidades: El Pensamiento Crítico efectivo requiere de habilidades mentales tales como el centrarse en la cuestión, analizar argumentos, clarificar, desafiar, observar y juzgar (Ennis, 1987). Aunque no existe un acuerdo común respecto a la cantidad y diversidad de las habilidades, un grupo de especialistas (APA, 1990) identificó las siguientes habilidades como centrales: interpretación, análisis, evaluación, inferencia, explicación y autorregulación.

c. Actitudes: Aunque la literatura habla sobre disposiciones (Norris, 1992; Ennis, 1987; Valenzuela y Nieto, 1997) el concepto más amplio resulta ser el de actitud. Las tendencias, propensiones, susceptibilidades, motivaciones, son factores que condicionan el actuar y por lo tanto la ejecución del Pensamiento Crítico. Para Ennis (1987) las disposiciones pueden lograrse ejecutando habilidades cognitivas concretas. Otros autores (Facione y Facione, 1992) consideran las disposiciones como hábitos mentales. En general existe una amplia aceptación al hecho de que la Motivación es un factor clave para el desencadenamiento del pensamiento crítico. 


\section{Investigación como estrategia pedagógica}

La investigación como estrategia pedagógica requiere promover espacios de inclusión, de diversidad y de creatividad del docente, para aprovechar una pregunta del niño en una investigación futura; favorece en el docente la pasión por enseñar, por potenciar las habilidades y destrezas de los niños, para aprovechar la capacitación, las oportunidades y la recreación, en lugar de reprimir o castigar (Alfaro, 2015).

\section{Metodología}

La investigación se desarrolló en la Institución Educativa Celinda Mejía López de Barroblanco ubicada en el municipio de Santa Ana, Magdalena, en el mes de noviembre del 2017. Se realizaron 45 encuestas a los estudiantes del grado sexto, para recopilar la información necesaria se emplearon la observación, la encuesta y la entrevista. Se describe una metodología de tipo descriptivo cualitativo.

Las técnicas empleadas en el proceso investigativo son:

- La observación: Es construir un bosquejo del problema y recolectar datos. Según Scribano (2007) en esta el observador y/o los observadores toman roles activos durante la situación, relaciones interpersonales y/o prácticas que se han estado observando y contextos.
- La encuesta: Esta técnica es aplicada a estudiantes para conocer y obtener información sobre la variable de habilidades del pensamiento crítico obteniendo resultados cualitativos de la muestra; para responder una encuesta acerca de las formas y estrategias que llevan a cabo para aprender, y las dificultades que se le presentan para ello (Martínez, 2011) (tabla 1).

- Entrevista: En la metodología cualitativa, la entrevista constituye encuentros reiterados, cara a cara entre el investigador y el entrevistado, con la finalidad de conocer la opinión y la perspectiva que tiene respecto a sus experiencias o situaciones vividas (Taylor y Bogdan, 1992).

\section{Resultados}

Ser realizaron unas encuestas a los estudiantes de sexto grado (tabla 1), tuvieron como fin dar a conocer las dificultades que ellos presentan al momento de trabajar con los textos en clase, esta técnica mostro que los estudiantes culpan a la profesora por no presentar estrategias para leer y algunos estudiantes demostraron evasión a la pregunta formulada.

En la tabla 1 se hace el análisis con gráficos y descripción de los resultados de las encuestas realizadas a los estudiantes partiendo de la categorización de las preguntas.

TABLA 1

Encuesta realizada a los estudiantes

\begin{tabular}{ll}
\hline Categorías & \multicolumn{1}{c}{ Subcategorías } \\
\hline & Tipo de texto (A. textos densos y profundos, B. textos de fácil y rápida lectura, C. \\
Pmbos tipos, D. no sabe no responde) & \\
de lectura & Tipo de lectura (A. poesía, B. teatro, C. narrativa, D. libros científicos, E. periódicos, \\
& $\begin{array}{l}\text { F. revistas técnicas, G. ensayos, H. revista de entretenimiento, I. obras literarias, J. } \\
\text { deportivas, K. otros. }\end{array}$ \\
\hline
\end{tabular}

Fuente: elaboración propia. 


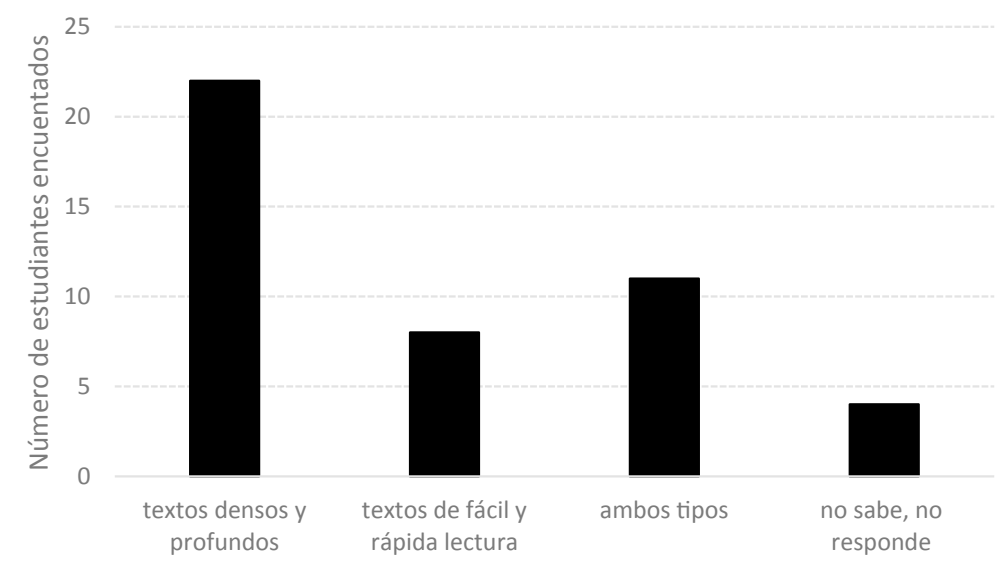

Figura 1. Tipo de texto

Fuente: elaboración propia.

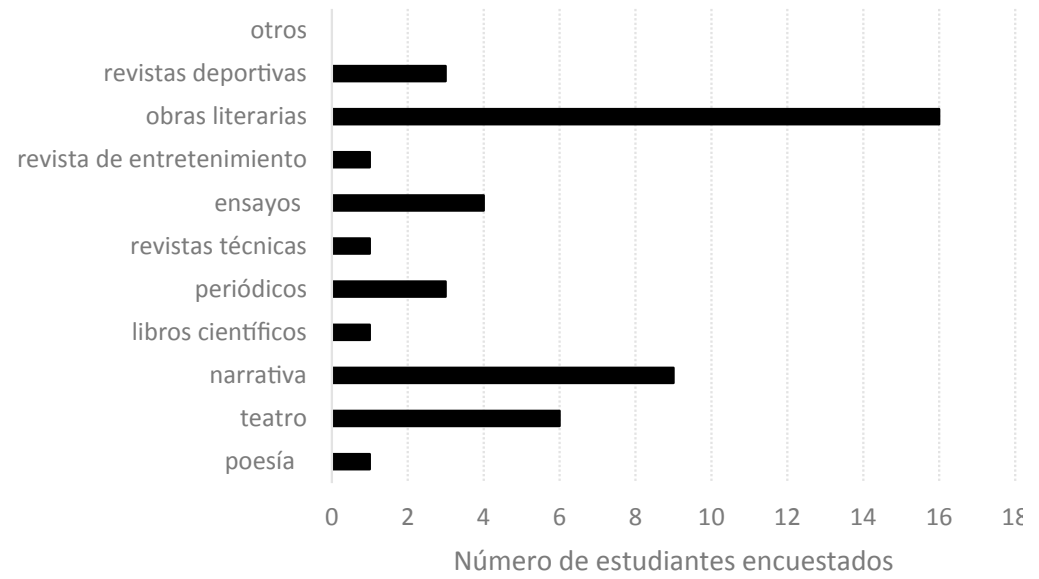

Figura 2. Tipos de lectura

Fuente: elaboración propia.

Con respecto a la preferencia de lectura de los jóvenes, la encuesta arrojo los resultados expuestos en la figura 1.

Como se ve en la Figura 1, los estudiantes prefieren leer textos variados, aunque los textos densos y profundos tuvieron mayor acogida siendo la opción más marcada por los estudiantes al momento de realizar la encuesta; debemos recordar que las escuelas deben fomentar la lectura variada y guiada a cumplir objetivos claves en la enseñanza de los estudiantes.
Sobre el gusto que mantienen los estudiantes al momento de leer, se observa a las obras literarias por encima de las demás, es importante que los estudiantes se sientan cómodos con lo que leen porque facilitara la transición de conocimientos y la misma activación de conocimientos previos permitiendo así una comprensión total del texto (figura 2). La narrativa tubo la segunda mayor acogida mientras que el teatro ocupo un tercer lugar. 
Análisis de la categorización a la entrevista de la docente

\section{Espacio de lectura crítica}

En esta categoría se pudo evidenciar que la docente si da espacios en sus horas de clase a la lectura, según lo dicho por la docente el día cinco (5) de noviembre manifestó en la entrevista las siguientes palabras: "sacamos un espacio en la semana para trabajar textos de comprensión lectora con mira a fortalecer su proceso lectoescritor". Aunque en diferentes observaciones y conversaciones con los estudiantes, se percató que en ocasiones no se da este espacio, por ende, es una de las causas por la que los estudiantes presentan falencias en la lectura, más específicamente en el nivel crítico.

\section{Estrategias de lectura crítica}

La profesora declaro que una de las estrategias que más utiliza es la lectura guiada con libro, talleres y mesa redonda. Esto se puede ver en lo dicho textualmente por ella "Recurso... bueno generalmente los talleres son más que todo los que se utilizan para poder fomentar en ellos la parte crítica o puesta en común o mesas redondas". Como lo afirmó en la entrevista estas actividades son llevadas a cabo en el aula de clases. Por eso, la docente debería de cambiar de estrategias e implementar nuevas actividades para promover la lectura crítica, por tal motivo se concluye que hace falta de manera permanente la aplicación de actividades en lectura crítica que conlleven a mejorar y fortalecer el proceso de aprendizaje, ya que, la comprensión lectora debe ser un proceso activo donde los estudiantes relacionen los conocimientos previos y la información del texto construyendo así nuevos saberes.

\section{Conocimientos sobre la lectura crítica}

Para la docente es importante desarrollar competencias a nivel de lectura según lo dicho por ella "permiten desenvolver al estudiante en cualquier área del conocimiento", se puede notar un gran interés por este tema. Por eso se pudo observar que tiene un conocimiento previo sobre el tema y es consciente de que la lectura crítica ayuda a los estudiantes a mejorar su desempeño académico, no solo en su área si no en cualquiera que manejen esto. En definitiva, es necesaria la comprensión sobre que es la lectura crítica, sobre esto Cassany afirma que "la lectura crítica es la más exigente y compleja que nos podamos imaginar, porque requiere un nivel de interpretación más elevado, así como habilidades y conocimientos previos para realizarla.".

\section{Valoración de lectura crítica}

La profesora afirma en la entrevista lo siguiente, "Los reconozco obviamente (risa) como docente de lengua castellana debemos conocerlos", además hace una valoración de los estudiantes afirmando que estos se encuentran en un nivel básico de lectura. Por ende, se presentó una pregunta asociada con la valoración hacia los estudiantes teniendo en cuenta los niveles de lectura crítica, aunque en la respuesta se pudo notar que la docente se mantuvo esquivo referente al tema, dando a entender que no maneja con totalidad estos niveles que ayudan al desarrollo del pensamiento del educando. Para Paul y Ender (2003), "Existe un cierto número de niveles de lectura cuidadosa. Aquî destacamos Cinco de ellos: parafrasear, explicar, analizar, evaluar y representar". Donde podemos notar que se comienza por la interpretación del lector hasta entender las del autor. 
Leer es un acto que permite al hombre desarrollarse no solo como una persona competente sino como ser humano, debemos comprender que las ventajas de la lectura son muchas y debemos dedicarle tiempo a la comprensión de los textos, por lo mismo es necesario que se usen métodos, estrategias que abran paso a la motivación y los intereses del lector. Para comprender e interpretar textos, se requiere una serie de conocimientos que se inician desde muy temprano en las escuelas, comenzando desde las vocales, unión de palabras, frases completas hasta la culminación de los grados con identificación de ideas en un texto, interpretación y reseña de texto, etc. Todo se debe poner en práctica para que, al momento de leer, se convierta en un acto de regocijo en el que se desarrollen múltiples dimensiones del ser humano.

El grupo investigador desarrolló actividades con el objetivo de establecer estrategias para el desarrollo de la lectura crítica en los estudiantes de décimo grado de la Escuela Normal Superior La Hacienda, enfocándonos en los tres niveles de comprensión lectora (literal, inferencial y critico), soportados por los múltiples enfoques de investigadores y los lineamientos curriculares.

Para poder cumplir con el objetivo, se desarrolló un proceso de diagnóstico e identificación de problemática, con el cual se usó una serie de recursos como lo fue la observación, tres talleres diagnósticos, encuesta a estudiantes y entrevista a la docente, donde se evidencio que con respecto a los niveles de lectura, los estudiantes presentan dificultades constantes en los niveles literales e inferenciales pero en el que más bajo reconocimiento presentan es el crítico, por lo tanto se decidió realizar múltiples estrategias de lectura basadas en conceptos de Solé (1998) y enfocando la importancia en el desarrollo de las habilidades de los estudiantes al momento de comprender los textos, además, de promover el hábito lector y dar una nueva orientación a los jóvenes al momento de leer.

\section{Discusión}

Mediante los resultados obtenidos se hace pertinente y necesario fortalecer las estrategias que se han estado implementando para incentivar la lectura en los estudiantes, es necesario identificar los pros y contras que tiene la asignación de lecturas muy densas para su comprensión al inicio del proceso. Es recomendable iniciar por las lecturas más sencillas y cortas para acercar el hábito a los estudiantes.

A fin de mejorar los procesos lectores en los estudiantes se recomienda:

- Mejorar el desempeño del docente en cuanto a desarrollar estrategias en las diversas áreas.

- Motivar al estudiante con diferentes actividades atendiendo a los intereses y necesidades de los estudiantes de acuerdo con las edades, sexo, y demás factores concernientes

- Se recomienda que de manera inmediata o a corto plazo se realice la aplicación de la propuesta que consiste en actividades para la lectura crítica.

- Se sugiere que la autoridad intervenga para que los docentes participen de manera masiva y multiplicadora en la aplicación de este proyecto. 


\section{Referencias}

Alfaro, C. (2015). La investigación como estrategia pedagógica en la Guajira, desde una perspectiva de la inclusividad y diversidad como nuevo paradigma para el desarrollo de habilidades, destrezas y competencias. Educación y ciudad, (29), 150-162. Recuperado de https://dialnet.unirioja.es/descarga/articulo/5704941.pdf

Almeida, M., Coral, F., Ruiz, M. y Grisales, M. (2014). Didáctica Problematizadora para la configuración del Pensamiento Crítico en el marco de la atención a la diversidad. [Tesis de pregrado]. Universidad de Manizales, Manizales, Colombia. Recuperado de http://ridum.umanizales.edu.co:8080/ xmlui/handle/6789/1727

Arrieta, M. (2016). Los masones en el mundo: Geopolítica masónica. La historia de los hijos de la luz. Córdoba: Almuzara.

APA. (1990). Critical Thinking: A Statement of Expert Consensus for Purposes of Educational Assessment and Instruction. The Delphi Research Report. American Philosophical Association. Recuperado de http://www.insightassessment.com/dex.html

Bailin, S., Case, R., Coombs, J. y Daniels, L. (1999). Conceptualizing critical thinking. Journal of curriculum studies, 31(3), 285-302. Recuperado de http://www.iskconeducation.org/

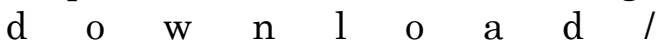
BAILIN,CASE,COOMBS $\% 20 \& \% 20$ DANIELS\%20-\%20Conceptualizing\%20critical\%20thinking.pdf

Baqueriza, C. y Anchundia, O. (2013). Aplicación de lectura crítica en los procesos de enseñanza-aprendizaje para los estudiantes de segundo año especialización lengua y literatura. Pro- puesta guía de métodos andragógicos. [Tesis de posgrado]. Universidad de Guayaquil, Guayaquil, Ecuador. Recuperado de http://studylib.es/ doc/3674627/tesis-carmen-baquerizo. pdf

Bierman, A. y Assali, R., (1996). The critical thinking handbook. Upper Saddle River, NJ: Prentice Hall.

De Zubiría, J. (2014). Las razones del bajo desempeño en las pruebas PISA. El tiempo. Recuperado de http://www.eltiempo.com/vida-de-hoy/educacion/ las-razones-delbajo- desempeno-enlas-pruebas-pisa_13789475-4

Ennis, R. H. (1987). A taxonomy of critical thinking dispositions and abilities. En J. B. Baron, y R. J. Sternberg (Eds.), Teaching Thinking Skills. New York, USA: Freeman and Company.

Facione, P y Facione, N. (1992). The California Critical Thinking Dispositions Inventory (CCTDI); and the CCTDI Test Manual. Millbrae, CA: The California Academic Press. Fowler, B. (1997). Longview Community College, Critical thinking Across the Curriculum Project, Critical Thinking Definitions. Recuperado de http:// isites.harvard.edu/fs/docs/icb.topic204027.files/Critical_thinking_d efinitions.pdf.

González, M. (2008). Competencias Genéricas y formación profesional: un análisis desde la docencia universitaria. Revista Iberoamericana de educación. 47(1). 185-209. Recuperado de https://dialnet.unirioja.es/servlet/ articulo?codigo $=2736795$ pae

Halpern. (1998). Knought and Knowledge: An introduction to critical thinking. Mahwah: Lawrence Erlbaum. https://doi.org/10.1002/(SICI)1099$0720(199704) 11: 2<179$ :: A ID ACP453>3.0.CO;2-S 
López, G. (2012). Pensamiento crítico en el aula. Docencia e Investigación, 22. 41-60. Recuperado de http://www.educacion.to.uclm.es/pdf/revistaDI/3_22_2012.pdf

López, L. (2011). La Clase para Pensar. Editorial: Ediciones Uninorte.

Martínez, J. (2011). Métodos de investigación cualitativa. Silogismo, (08). Recuperado de http://www.cide.edu.co/doc/ investigacion $/ 3 . \% 20$ metodos $\% 20$ de\%20investigacion.pdf

McPeck, J. (1981). Critical Thinking and Education (El Pensamiento crítico y la Educación). Oxford: St. Martin's Press.

Mendosa, P. (2015). La investigación y el desarrollo de pensamiento crítico en estudiantes universitarios. [Tesis Doctoral]. Universidad de Málaga, Málaga, Colombia.

Montoya, J. y Monsalve, J. (2008). Estrategias didácticas para fomentar el pensamiento crítico en el aula. Revista Virtual Universidad Católica del Norte. 1-25. Recuperado de https://www. redalyc.org/pdf/1942/194215513012. pdf

Morán, A. y Uzcátegui, A. (2006). Estrategias para el desarrollo de la comprensión lectora de los estudiantes del octavo grado de educación básica. $R e$ vista de Artes y Humanidades Unica, 7(16). Recuperado de http://www.redalyc.org/html/1701/170118726003/

Norris, S. (1992). Testing for the disposition to think critically. Informal Logic, 2(3), 157-164.

Páez, R. y Rondón, G. (2014). La lectura crítica: propuestas para el aula derivadas de proyectos de investigación educativa. Recuperado de http://biblioteca. clacso.edu.ar/Colombia/fce-unisalle/20170117041131/lecturacr.pdf
Paul, R. y Elder, L. (2003). La mini-guía para el pensamiento crítico. Conceptos y herramientas. Recuperado de h t t p : / / e s . c a l a m e o.com / $\mathrm{read} / 0006702414862 \mathrm{f} 2 \mathrm{~b} 53 \mathrm{cdc}$

Santiuste B., García, G., Ayala, C. y Barriguete, C. (1996). Procesos y estrategias de comprensión lectora, aplicación a la enseñanza de la Filosofía a través de textos. Madrid: Fugaz.

Scribano, A. (2007). El proceso de investigación social cualitativo. Buenos Aires: Prometeo Libros.

Serrano, S. (2014). La lectura, la escritura y el pensamiento. Función epistémica e implicaciones pedagógicas. Lenguaje, 42(1), 97-122. Recuperado de http://www.scielo.org.co/pdf/leng/ v42n1/v42n1a05.pdf

Serrano, S. (2008). El desarrollo de la comprensión crítica en los estudiantes universitarios. Hacia una propuesta didáctica. Educere, 12 (42). 505-514. Recuperado de http://www. scielo.org.ve/scielo.php?script=sci_ar $\mathrm{t} \quad \mathrm{t} \quad \mathrm{e} \quad \mathrm{x} \quad \mathrm{t} \quad \& \quad \mathrm{p} \quad \mathrm{i} \quad \mathrm{d}$ $=\mathrm{S} 1316-49102008000300011$

Solé, I. (1998). Estrategias de Lectura. Octava edición. Barcelona: Editorial Graó. Recuperado de https://media. utp.edu.co/referencias-bibliograficas/ uploads/referencias /libro/1142-estrategias-de-lecturapdf-N0aU6-libro. pdf

Tamayo, M. (2003). El proceso de la investigación científica ( $4^{\mathrm{a}}$ ed.). México, D.F.: Limusa.

Taylor, S. J y Bogdan, R.(1992). Introducción a los métodos cualitativos en investigación. La búsqueda de los significados. España: Paidós. 
Valenzuela, G. y Rochin, L. (2013). Libertad de expresión y su impacto en la democracia. Juridicas CUC, 9(1), 89100. Recuperado de
Valenzuela, J. y Nieto, A. (1997). Motivación y Pensamiento Crítico: Aportes para el estudio de esta relación. Revista Electrónica de Motivación y EmociónREME, 11(28). Recuperado de http:// reme.uji.es/articulos/numero28/article3/texto.html 